\title{
Influence of diabetes on endothelial cell response during sepsis
}

\author{
P. Schuetz $\cdot$ K. Yano $\cdot$ M. Sorasaki $\cdot$ L. Ngo • \\ M. St Hilaire • J. M. Lucas • W. Aird • N. I. Shapiro
}

Received: 12 October 2010 /Accepted: 7 January 2011 /Published online: 1 February 2011

(C) Springer-Verlag 2011

\begin{abstract}
Aims/hypothesis Several endothelial pathways of cell adhesion, coagulation and vascular endothelial growth factor (VEGF) signalling are activated during sepsis. The objective of this analysis was to investigate the influence of diabetes on biomarkers of endothelial cell activation in sepsis.

Methods This was a prospective observational cohort study of a convenience sample of adult patients (age $\geq 18$ years) for whom infection was clinically suspected and who presented to an urban tertiary care emergency department between February 2005 and November 2008. We investigated the association of diabetes and sepsis with various endothelial activation biomarkers of cell adhesion (E-selectin, vascular cell adhesion molecule 1 [VCAM-1] and intercellular adhesion molecule 1 [ICAM-1]), coagulation (plasminogen activator inhibitor 1 [PAI-1]) and VEGF signalling (soluble fms-like tyrosine kinase-1 [sFLT-1]).

Results A total of 207 patients (34\% with sepsis, 32\% with severe sepsis and $34 \%$ with septic shock) were studied, including 63 (30\%) with diabetes. Compared with patients without diabetes, patients with diabetes had significantly increased E-selectin and sFLT-1 levels overall; this was
\end{abstract}

P. Schuetz $(\bowtie) \cdot \mathrm{K}$. Yano $\cdot$ M. Sorasaki $\cdot \mathrm{L}$. Ngo $\cdot$ M. St Hilaire $\cdot$

J. M. Lucas $\cdot$ N. I. Shapiro

Department of Emergency Medicine,

Beth Israel Deaconess Medical Center,

1 Deaconess Road, CC2-W,

Boston, MA 02215, USA

e-mail: schuetzph@gmail.com

K. Yano $\cdot$ M. Sorasaki $\cdot$ W. Aird $\cdot$ N. I. Shapiro

Center for Vascular Biology Research, Harvard Medical School,

Boston, MA, USA most pronounced during septic shock in the stratified analysis. Multivariate models including age, sex, sepsis severity and other variables as potential covariates confirmed the association of diabetes with elevated circulating plasma levels of E-selectin (standardised $\beta 0.24, p<0.001$ ) and sFLT-1 (standardised $\beta 0.19, p<0.01$ ), but there was no significant association with VCAM-1, ICAM-1 or PAI-1. Conclusions/interpretation During septic shock, patients with diabetes had higher levels of circulating biomarkers of endothelial cell adhesion (E-selectin) and VEGF signalling (sFLT-1). Future studies should address whether enhanced activation of the endothelium places patients with diabetes at increased risk for the development of sepsis and worsening morbidity and mortality.

Keywords Coagulation · Diabetes $\cdot$ Endothelium . Inflammation
Abbreviations
APACHE II Acute Physiologic and Chronic Health
Evaluation II
BIDMC Beth Israel Deaconess Medical Center
$\mathrm{CHF} \quad$ Congestive heart failure
ED Emergency department
ICAM-1 Intercellular adhesion molecule 1
IQR
LPS Interquartile range
PAI-1 Plasminogen activator inhibitor 1
RAGE Receptor for advanced glycation endproducts
sFLT-1 Soluble fms-like tyrosine kinase-1
SIRS Systemic inflammatory response syndrome
SOFA Sequential organ failure assessment
VCAM-1 Vascular cell adhesion molecule 1
VEGF Vascular endothelial growth factor 


\section{Introduction}

More than 750,000 cases of severe sepsis are diagnosed each year in the USA, with an estimated mortality rate between $30 \%$ and $50 \%$ [1]. Despite significant advances in medical diagnosis and treatment, the overall survival in sepsis has not improved substantially over time [2, 3]. This can partly be explained by the fact that sepsis is not a disease, but a syndrome comprising heterogeneous pathophysiological entities. In addition, chronic co-morbid medical conditions may alter outcomes in sepsis and therefore play an important role when selecting sepsis treatments. A better understanding of the different pathophysiological entities and the influence of comorbid conditions is therefore a promising avenue of investigation for more targeted sepsis therapies and improved patient outcomes [4]. Diabetes is a major comorbid condition in septic patients because of its high prevalence and accompanying pathophysiological manifestations. Among patients with severe sepsis, around $10-30 \%$ are reported to have diabetes [5-7]. Yet the role of diabetes in sepsis is not completely understood.

Emerging evidence suggests that endothelial cell activation and dysfunction play an essential role in the pathophysiology of sepsis $[8,9]$. Previous work has suggested that the endothelium is critical to the vascular leak and the subsequent shock state that contributes to mortality among patients with sepsis $[2,10]$. In addition, the endothelium participates in the inflammatory response during sepsis through signalling molecules, such as E-selectin, which adheres to the circulating white blood cells to facilitate cell rolling, and soluble vascular cell adhesion molecule 1 (VCAM-1) and soluble intercellular adhesion molecule 1 (ICAM-1), which solidify cellular bonds for transmigration [11]. In addition, vascular endothelial growth factor (VEGF) contributes to vascular leak and propagation of host response and its soluble receptor (sVEGFR-1) is an anti-inflammatory peptide and inhibits VEGF activity [11]. In addition, sepsis-induced activation of the endothelium creates a net procoagulant response with an increase in molecules such as plasminogen activator inhibitor 1 (PAI-1) $[9,11]$. In previous work, we found increased levels of these endothelial biomarkers in septic patients and correlations with sepsis severity, organ dysfunction and sepsis outcomes [12].

Previous investigations have suggested that diabetic patients are particularly prone to endothelial dysfunction during sepsis because hyperglycaemia, insulin resistance and obesity activate different endothelial pathways in nonseptic conditions [13, 14]. Clinical studies have demonstrated increased levels of the adhesion markers E-selectin, ICAM-1 and VCAM-1 in patients with diabetes [15-17]. Increased levels of PAI-1 have also been found in patients with diabetes, increasing their risk for thrombotic disease $[18,19]$. Interestingly, these elevated levels of circulating markers are thought to play a role in the accelerated development of atherosclerotic plaques in patients with diabetes. Similarly, higher levels of VEGF and its receptor, sFLT-1, in patients with diabetes are thought to promote diabetic nephropathy and retinopathy [20]. However, it remains unclear whether diabetes alters the endothelial activation during sepsis. We therefore examined different endothelial biomarkers in a well-defined cohort of septic patients and sought to study the influence of pre-existing diabetes on the production of biomarkers associated with endothelial cell activation in sepsis.

\section{Methods}

Study design and setting This is a secondary analysis of a prospective observational cohort study of patients who presented to the emergency department (ED) of the Beth Israel Deaconess Medical Center (BIDMC), an urban academic teaching hospital, between February 2005 and November 2008. A more detailed description of the assembly of the original cohort is described elsewhere [12]. The aim of the analysis presented here was to investigate the influence of diabetes on endothelial markers mirroring endothelial activation and clinical outcomes in this patient cohort.

Selection of participants Eligible patients were 18 years of age or older and presented to the ED with clinical suspicion of an infection, as determined by the treating ED clinician. Patients were selectively enrolled in order to achieve a balanced distribution of different sepsis severities. Patients were classified as having diabetes if they or the medical chart indicated a past medical history of diabetes, regardless of duration or type of diabetes; $\mathrm{HbA}_{1 \mathrm{c}}$ or islet antibody levels were not assessed to confirm the diagnosis or type of diabetes. All patients were followed for the first $96 \mathrm{~h}$ of their hospital course or until hospital discharge.

The hospital ethics board and the committee for clinical investigations at BIDMC approved the study and all patients or their representatives provided written informed consent.

Data collection and processing We gathered baseline and sequential clinical information by structured patient or proxy interviews, bedside assessment by study nurses and standardised medical record reviews. Specifically, we recorded demographics, past medical history, co-morbid illness, suspected source of infection, vital sign information, physical examination findings and the results of laboratory and radiological testing. Serial blood draws were 
performed at time 0 and $24 \mathrm{~h}$, as available. Samples were drawn in EDTA tubes, centrifuged at 2,500 $\mathrm{g}$ at $4^{\circ} \mathrm{C}$, and frozen at $-80^{\circ} \mathrm{C}$ within $1 \mathrm{~h}$ of collection.

Method of measurement We assayed five biomarkers of different endothelial pathways: plasma levels of E-selectin, ICAM-1, VCAM-1 and PAI-1, with a multiplex panel (Multiplex Human Cardiovascular Disease Multiplex Panel 1 kits; Millipore, Billerica, MA, USA); and sFLT-1, which was assayed using the Quantikine ELISA kits (R\&D systems, Minneapolis, MN, USA). In addition, IL-6 was assayed using the Luminex 200 instrument (Human Cardiovascular Disease Multiplex Panel 3; Millipore). All assays were performed in duplicate and the average levels were used for analysis. In addition, we measured venous lactate levels, a marker of illness severity and tissue hypoperfusion at study enrolment.

Sepsis severity Within this study, we sought to examine the influence of diabetes on biomarker levels across the entire spectrum of sepsis syndromes. For this purpose, patients were characterised as having 'sepsis', 'severe sepsis' or 'septic shock' according to the modified criteria of the American College of Chest Physicians/Society of Critical Care Medicine sepsis syndromes [21]. The 'sepsis' category comprised patients with suspected infection with or without systemic inflammatory response syndrome (SIRS) criteria. The decision to combine these groups (with and without SIRS) was based on previous publications demonstrating no mortality difference based on SIRS criteria alone so that 'severity' is equivalent, as well as the SIRS criteria falling out of favour in the latest consensus guidelines. $[22,23]$. The presence and source of a suspected focal infection was classified as suspected lower respiratory tract infection, urinary tract infection, intra-abdominal infection, skin or soft tissue infection, or endocarditis. The clinical suspicion of infection was abstracted from the medical record by using criteria based on the medical decisionmaking section of the chart along with the diagnosis assigned by the treating physicians. 'Severe sepsis' was defined as sepsis with concomitant organ dysfunction defined by meeting one or more of the following organ dysfunction definitions: central nervous system, new altered mental state and/or new onset of Glasgow coma score $<15$; respiratory, any mechanical ventilation, supplemental oxygen required to maintain oxygen saturation $>95 \%$ and/or respiratory rate $>24$ beats per min; cardiovascular, any vasopressor use, systolic blood pressure $<90 \mathrm{mmHg}$ after $20 \mathrm{ml} / \mathrm{kg}$ bolus; renal, urine output $<0.5 \mathrm{ml} \mathrm{kg}^{-1} \mathrm{~h}^{-1}$, creatinine $>50 \%$ of baseline or $>2.0$ if baseline is unknown; hepatic, aspartate aminotransferase/alanine aminotransferase $>80$ (new); haematopoietic, platelet count $<100,000$ and/or prothrombin time/partial thromboplastin time $>50 \%$ of normal; or metabolic, lactate $>2.5 \mathrm{mmol} / \mathrm{l}$. 'Septic shock' was defined as sepsis complicated by hypotension (SBP $<90 \mathrm{mmHg}$ after a $20-30 \mathrm{ml} / \mathrm{kg}$ fluid challenge) [22].

Clinical outcomes To assess organ dysfunction, we calculated sequential organ failure assessment (SOFA) score as a validated continuous score for organ failure. [24] The Acute Physiologic and Chronic Health Evaluation II (APACHE II) score was used as a measure of disease severity, based on worst vital signs, as originally described by Knaus et al. [25] We further assessed mortality within this cohort, which was defined as death of any cause until hospital discharge.

Statistical analysis First, levels of endothelial markers in patients with and without diabetes were compared, stratified by sepsis syndrome, using Wilcoxon-Mann-Whitney $U$ tests. Next, to obtain the independent association of diabetes with the markers, we performed multivariate linear regression analysis with diabetes as the predictor (independent covariate) and each biomarker as the outcome (dependent). We further constructed multivariable linear regression models with stepwise forward selection (significance criterion for entry into the model, $p<0.2$; significance criterion for staying in the model, $p<0.05$ ) of variables using the following candidate covariates: diabetes, sepsis syndrome, age, sex, weight, comorbidities (congestive heart failure $[\mathrm{CHF}]$, chronic renal failure, chronic obstructive pulmonary disease and cancer). Logtransformed biomarker levels were normally distributed. We report standardised $\beta$ s ( $\beta$ coefficients) from the linear regression models. Because the $\beta$ coefficients are all measured in standard deviations, instead of the units of the variables, they can be compared with one another. Next, we investigated the association of diabetes with organ failure, defined by the SOFA score [24]. We modelled the association of diabetes with SOFA in a multivariate linear regression analysis and used stepwise forward selection procedures with the same candidate covariates as outlined above.

All reported CIs are two-sided $95 \%$ intervals and tests were performed at the two-sided 5\% significance level. All analyses were performed with SAS 9.2.

\section{Results}

Study cohort characteristics There were a total of 207 patients in the study cohort, of which $63(30 \%)$ had preexisting diabetes. There was a relatively even distribution of sepsis severities: 70 patients (34\%) had sepsis; 66 (32\%) had severe sepsis; and 71 (34\%) had septic shock. The source of infection was community-acquired pneumonia in $26 \%$, skin infection in $28 \%$, urinary tract infection in $13 \%$, 
wound infection in $3 \%$ and other infections in the remaining $30 \%$ of patients. Patients with diabetes were older, with a higher proportion of renal impairment, $\mathrm{CHF}$ and severe sepsis syndromes compared with patients without diabetes (Table 1).

Association of diabetes with endothelial biomarkers Overall, patients with sepsis and diabetes had significantly increased median levels of E-selectin (71.4 [interquartile range $\{\mathrm{IQR}\}$ 45.8-116.0] vs 45.1 [IQR 30.1-68.6], $p<0.0001)$ and sFLT-1 (192 [IQR 103-378] vs 112 [IQR 70-187], $p<0.0001)$. The levels of ICAM-1 $(p=0.4)$, VCAM-1 $(p=0.15)$ and PAI-1 $(p=0.51)$ were similar in diabetic and non-diabetic patients. To investigate whether an increase in circulating levels of E-selectin and sFLT-1 was found across sepsis syndromes, we performed a stratified analysis and compared biomarker levels in patients with diabetes and without diabetes within the three sepsis syndromes (sepsis, severe sepsis and septic shock). Thereby, we found significantly higher median levels of E-selectin (119 [IQR 71-168] vs 61 [IQR 37-83], $p<0.001)$ and sFLT-1 (370 [IQR 243-712] vs 190 [IQR 113-351], $p=0.001)$ in diabetic patients with septic shock; in all other sepsis syndromes and for the other endothelial biomarkers there was no difference in biomarker levels between patients with diabetes and without-diabetes (Fig. 1).

Next, to investigate whether there was an independent association of diabetes with levels of circulating biomarkers, we performed multivariate linear regression analysis using a forward stepwise selection procedure. In a model with the biomarker as the dependent variable, and diabetes, sepsis syndrome, age, sex, weight, $\mathrm{CHF}$ and chronic renal failure as potential predictors, we found a significant independent association of diabetes with circulating levels of E-selectin (standardised $\beta 0.24, p=0.0002$ )

Table 1 Patient characteristics

Values are expressed as median (IQR) or number (\%)

$p$ value corresponds to the comparison of patients with diabetes and without diabetes COPD, chronic obstructive pulmonary disease; $\mathrm{WBC}$, white blood cell

\begin{tabular}{|c|c|c|c|c|}
\hline Variable & Total $(n=207)$ & No diabetes $(n=144)$ & Diabetes $(n=63)$ & $p$ value \\
\hline \multicolumn{5}{|l|}{ Demographics } \\
\hline Age & $58(46-74)$ & $57(43-74)$ & $64(54-74)$ & 0.02 \\
\hline Female sex & $108(52)$ & $76(53)$ & $32(51)$ & 0.75 \\
\hline Race & & & & 0.07 \\
\hline White & $159(77)$ & $113(78)$ & $46(73)$ & \\
\hline African-American & $26(13)$ & $14(10)$ & $12(19)$ & \\
\hline Other & $22(10)$ & $17(12)$ & $5(8)$ & \\
\hline \multicolumn{5}{|l|}{ Comorbidities } \\
\hline COPD & $16(8)$ & $10(7)$ & $6(10)$ & 0.52 \\
\hline $\mathrm{CHF}$ & $29(14)$ & $14(10)$ & $15(24)$ & 0.007 \\
\hline Renal disease & $17(8)$ & $3(2)$ & $14(22)$ & $<0.0001$ \\
\hline Cancer & $38(18)$ & $29(20)$ & $9(14)$ & 0.31 \\
\hline Sepsis syndrome & & & & 0.02 \\
\hline Sepsis/infection & $70(34)$ & $57(40)$ & $13(21)$ & \\
\hline Severe sepsis & $66(32)$ & $43(30)$ & $23(37)$ & \\
\hline Septic shock & $71(34)$ & $44(30)$ & $27(43)$ & \\
\hline \multicolumn{5}{|l|}{ Severity of disease } \\
\hline SOFA score & $2(0-5)$ & $1(0-4)$ & $4(1-6)$ & $<0.0001$ \\
\hline APACHE II score & $11(6-19)$ & $9(5-17)$ & $15(8-22)$ & $<0.0001$ \\
\hline Lactate & $1.49(1.01-2.40)$ & $1.37(0.97-2.12)$ & $2.07(1.33-3.00)$ & $<0.0001$ \\
\hline Initial glucose level $(\mathrm{mmol} / \mathrm{l})$ & $6.9(5.7-8.8)$ & $6.4(5.5-7.7)$ & $9.9(7.4-13.3)$ & $<0.0001$ \\
\hline \multicolumn{5}{|l|}{ Inflammatory markers } \\
\hline WBC $\left(\times 10^{9} / 1\right)$ & $11.8(8-16.4)$ & $11.6(7.8-15.5)$ & $12.8(8.6-18.1)$ & 0.14 \\
\hline IL-6 (pg/ml) & $91(30-327)$ & $80(32-260)$ & $172(78-1350)$ & $<0.0001$ \\
\hline \multicolumn{5}{|l|}{ Endothelial markers } \\
\hline E-selectin (ng/ml) & $49.2(33.0-79.4)$ & $45.1(30.0-68.6)$ & $71.4(45.7-116)$ & $<0.0001$ \\
\hline VCAM-1 $(\mu \mathrm{g} / \mathrm{ml})$ & $1,120(907-1,430)$ & $1,120(893-1,415)$ & $1,155(973-1,790)$ & 0.15 \\
\hline ICAM-1 (ng/ml) & $176(136-262)$ & $176(137-262.5)$ & $186(129-286)$ & 0.38 \\
\hline PAI-1 (ng/ml) & $409(262-791)$ & $38.8(26.2-72.7)$ & $54.5(29.1-107)$ & 0.051 \\
\hline sFLT-1 (pg/ml) & $117(69-208)$ & $112(70-187)$ & $192(103-378)$ & $<0.0001$ \\
\hline
\end{tabular}


Fig. 1 Biomarker levels in patients with diabetes and without diabetes and different severities of sepsis:

(a) E-selectin; (b) VCAM-1;

(c) ICAM-1; (d) PAI-1; and

(e) sFLT-1. Without diabetes, grey bars; with diabetes, black bars. Vertical bars represent $95 \%$ CIs. ${ }^{*} p<0.05$ a

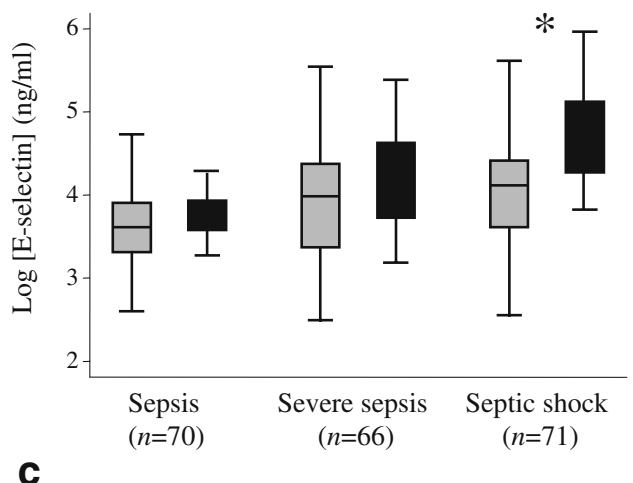

C

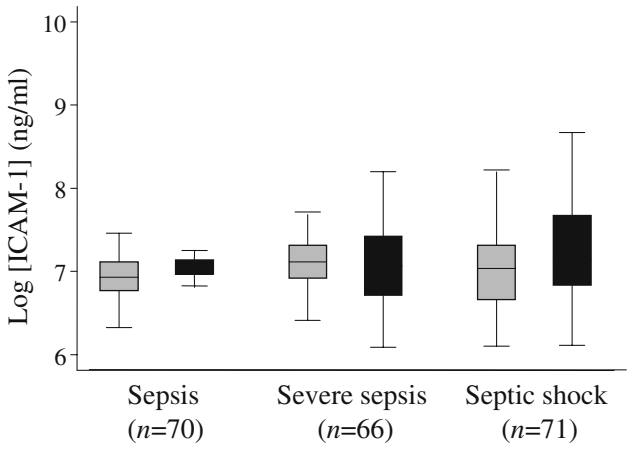

e

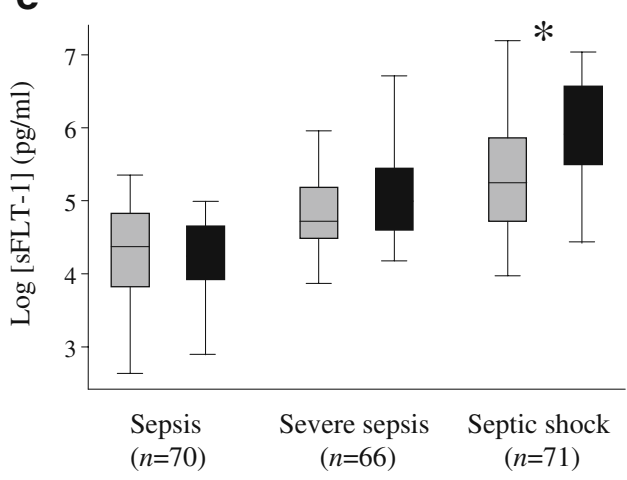

b

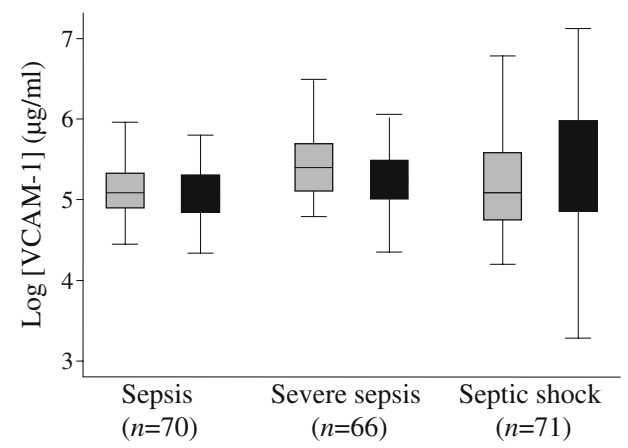

d

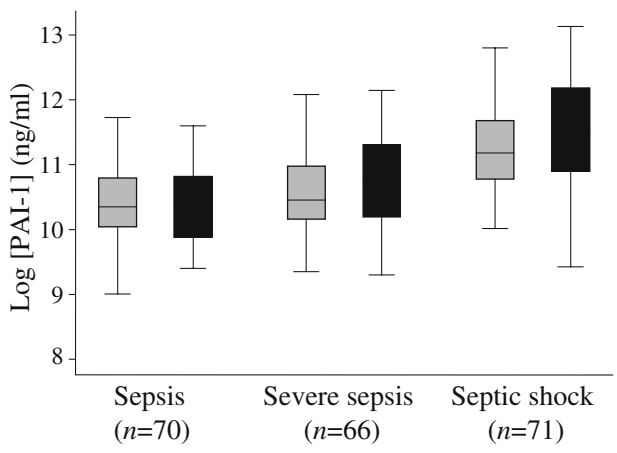

and sFLT-1 (standardised $\beta$ 0.19, $p=0.0011$; Table 2). For ICAM-1, VCAM-1 and PAI-1, diabetes was not significantly associated with the circulating biomarker level.

Association of blood glucose levels and endothelial biomarkers In univariate linear regression analysis, initial glucose levels showed a significant association with circulating levels of E-selectin (standardised $\beta$ 0.32, $p<0.0001$ ), sFLT-1 (standardised $\beta$ 0.31, $p<0.0001$ ), ICAM-1 (standardised $\beta$ 0.17, $p=0.02$ ), VCAM-1 (standardised $\beta 0.34, p<0.0001$ ) and PAI-1 (standardised $\beta 0.24$, $p<0.001$ ). To investigate whether this association was also true when including severity (sepsis syndrome), diabetes, age, sex, weight, comorbidities (CHF, chronic renal failure, chronic obstructive pulmonary disease and cancer) as potential covariates, we performed multivariate regression analysis.
In this analysis, the glucose level was not significantly associated with any of the five endothelial markers.

Association of diabetes with sepsis severity and organ failure Next, we investigated the association of diabetes with sepsis severity (defined by sepsis syndrome) and organ dysfunction (SOFA score). In our sample, diabetic patients tended to have more severe sepsis syndromes compared with patients without diabetes. Patients with diabetes had higher SOFA scores on admission (4 [1-6] vs $1[0-4], p<0.0001)$ and in univariate analysis, diabetes was significantly associated with higher SOFA scores (standardised $\beta 0.25, p<0.001$ ). This association of diabetes and higher SOFA score was also true when adjusted for sepsis syndrome and other significant confounders including age, sex and chronic renal failure in 
Table 2 Results of multivariable linear regression: association of diabetes with E-selectin and sFLT-1 levels

\begin{tabular}{lcccc}
\hline Predictor & \multicolumn{2}{l}{ Association of diabetes and E-selectin } & & \multicolumn{2}{l}{ Association of diabetes and sFLT-1 } \\
\cline { 2 - 3 } & Standardised $\beta(\mathrm{SE})$ & $p$ value & Standardised $\beta$ (SE) & $0.19(0.11)$ \\
\hline Diabetes & $0.24(0.09)$ & 0.0002 & 0.0011 \\
Sepsis syndrome & $0.35(0.05)$ & $<0.0001$ & $0.06)$ & $<0.0001$ \\
\hline
\end{tabular}

The table shows the association from multivariate linear regression analysis of diabetes on levels of E-selectin and sFLT-1 using stepwise forward selection and the following candidate covariates: diabetes, sepsis syndrome, age, sex, weight, comorbidities (CHF, chronic renal failure, chronic obstructive pulmonary disease and cancer); diabetes shows a significant association with both E-selectin and sFLT-1

multivariate linear regression analysis (adjusted standardised $\beta 0.09, p=0.006$ ).

\section{Discussion}

With this study, our primary objective was to investigate the effect of diabetes on different markers of endothelial activation during sepsis. We found significantly increased levels of E-selectin, a soluble leucocyte adhesion molecule, and sFLT-1, a VEGF receptor, in diabetic patients compared with patients without diabetes during the most severe stages of sepsis, namely septic shock. This difference was independent of initial glucose levels. For the other endothelial biomarkers under investigation (ICAM-1, VCAM-1 and PAI-1), no difference was observed. This finding suggests that patients with diabetes show a more pronounced activation of some endothelial pathways in sepsis, especially during more severe disease.

Prior studies have shown that several endothelial pathways activated during sepsis may also be upregulated in non-infected patients with diabetes. Subclinical tissue injury and increased fat mass lead to increased levels of pro-inflammatory cytokines and induce an inflammatory cascade at the site of the endothelium. Hyperglycaemia, insulin resistance, obesity, oxidative stress, as well as NEFAs, oxidised LDL-cholesterol, and advanced glycosylation endproducts are other factors known to interfere with cell adhesion molecules, proinflammatory molecules, coagulation and VEGF signalling at the endothelial wall in patients with diabetes $[13,14,17,26]$. Clinical studies have found increased levels of E-selectin, ICAM-1 and VCAM-1 in patients with diabetes, and higher levels were associated with a subsequent risk for cardiovascular events [16, 27, 28]. Similarly, high concentrations of PAI-1 and subsequent downregulation of fibrinolysis have been implicated in the increasing morbidity, mortality and risks for thrombotic disease in diabetic patients [18, 19]. VEGF and sFLT-1 may also play a role in the pathophysiology of atherosclerosis by initiating and propagating angiogenesis, and thereby providing a passage for inflammatory cells to the site of injury [29]. Increased concentrations of VEGF and sFLT-1 have been reported in patients with diabetes, and they may play a role in the development of diabetic nephropathy and retinopathy [20]. Thus, in summary, there is strong evidence from preclinical and clinical studies for concurrent activation of several endothelial pathways in patients with diabetes that are also upregulated during sepsis. However, it is largely unknown whether the upregulation of endothelial markers in non-infected patients with diabetes would affect their endothelial response during sepsis.

A recent analysis looked at several biomarkers of the coagulation system, including PAI-1 and inflammatory markers, in patients with septic shock from the Recombinant Human Protein C Worldwide Evaluation in Severe Sepsis (PROWESS) study [6]. The authors found similar short- and long-term mortality rates in patients with and without diabetes and no association of diabetes with the different biomarkers. Our study was concordant with these findings as PAI-1 levels were similar in diabetic and nondiabetic patients. One may postulate that our finding, that PAI-1 levels were similar in patients with diabetes and without diabetes, may potentially be explained by the fact that sepsis provides a much stronger systemic stimulus than the relative smaller derangements attributable to co-existent diabetes. Interestingly, there was also no association with diabetes and circulating levels of ICAM-1 and VCAM-1, although these are not specific to the endothelium [9]. However, we did find a more pronounced activation of the endothelium-specific adhesion molecule E-selectin in patients with diabetes, particularly during septic shock. We also found higher levels of sFLT-1, the origin of which has not yet been firmly established.

Another recent study investigated the profile over time of a number of different inflammatory cytokines, E-selectin, VCAM-1 and ICAM-1, in 19 diabetic patients compared with 23 non-diabetic healthy controls after injection of Escherichia coli lipopolysaccharide (LPS) [30]. Interestingly, within this small but well-controlled study, type 2 diabetes was associated with less pronounced LPS-induced increases in VCAM-1 and ICAM-1 and there was a trend towards an attenuated upregulation of E-selectin in patients with diabetes. In contrast to these results, we did not find any differences in VCAM-1 and ICAM-1 levels, and found 
higher E-selectin levels in diabetic patients with septic shock. Although the differences in results cannot be conclusively explained, we speculate that differences in LPS-induced upregulation vs naturally occurring infectioninduced upregulation and patient and infection-type heterogeneity, which includes a heavy comorbid burden within our study, may be important factors in this respect.

It is a matter of controversy whether diabetic patients respond differently to sepsis. Interestingly, some reports indicate an increased incidence of infections and higher mortality rates among patients with diabetes [5, 31, 32]. Others have found no difference in outcomes, or even improved outcomes in patients with diabetes [6, 33-35]. A recent epidemiological study found lower rates of organ dysfunction in diabetic patients with sepsis using data from 12.5 million septic patients included in the National Hospital Discharge Survey (USA) [33]. Within our cohort, patients with diabetes had higher sepsis severity and worse outcomes, in terms of organ dysfunction, compared with patients without diabetes. This was also true when adjusting for important confounders such as age, renal impairment and CHF. The discrepancies among these studies are likely due to various issues relating to the methods used: heterogeneity of the study population, the variability of infection type and severity included in different trials, differences in the definition of 'sepsis', incomplete information gathering regarding diabetes, incomplete follow-up information and potential selection bias.

Our study has a number of limitations. We assessed endothelial dysfunction solely by means of plasma biomarkers and do not have other measures of direct vascular physiology. A previous study comparing endothelial biomarkers with skin biopsies suggested that caution is required in the interpretation of these markers in plasma, which does not necessarily reflect the in situ activation state of endothelium [36]. Diabetes diagnoses were based solely on documentation in patients' medical records and were not confirmed using $\mathrm{HbA}_{1 \mathrm{c}}$ measurements. We did not differentiate diabetic patients based on the length of time they had had diabetes or the severity of their diabetes. Furthermore, the possibility that our 'non-diabetes' group included patients with undiagnosed diabetes cannot be ruled out. Our numbers, while relatively large compared with other studies investigating endothelial biomarkers, are still too small to draw definitive conclusions. Finally, the population was selected to obtain a spectrum of sepsis severities as opposed to a consecutive sample of patients, exposing us to selection bias. Our sepsis patient group comprised patients with clinical suspicion of infection, with and without positive SIRS criteria, because previous studies - including one in our institutionshowed the inherent limitations of these criteria [22]. We found no differences in levels of any of the assessed markers in sepsis patients with and without at least two SIRS criteria.
Additionally, there may have been patients with potential infection who were not included in this study and other patients without infection who were still included in the sepsis group.

Previous studies suggested that the endothelium plays a critical role in the pathogenesis of the vascular leak, ultimately leading to shock and thereby contributing to the unacceptably high mortality among patients with sepsis [10] [2]. A better understanding of the function of the endothelium in sepsis, and of the influence of diabetes and other comorbidities on endothelium activation may one day lead to new life-saving therapies [2]. We believe that our study has advanced the understanding of diabetes and markers of endothelial activation in different severities of sepsis and is thus noteworthy. Importantly, the association of diabetes and the endothelium may also be influenced by other factors such as the type I transmembrane receptor for advanced glycation endproducts (RAGE), which interacts with cytokine-, LPS-, oxidised LDL- and glucose-triggered cellular reactions [37]. Thereby, it turns a short-lasting inflammatory response into a sustained change of cellular function driven by perpetuated activation of proinflammatory transcription factors [37]. Future studies should address whether there is an association between endothelial cell activation with oxidative stress markers and/or RAGE plasma levels.

In conclusion, our data suggest that during the later and most severe stages of sepsis, patients with diabetes show a more pronounced activation of endothelial pathways, as represented by moderately increased circulating levels of E-selectin and sFLT-1. Future studies are needed to address whether more pronounced activation of endothelial cell markers places patients with diabetes at an increased risk of developing sepsis and, during sepsis, whether they are more likely to suffer from organ dysfunction and worse outcomes.

Acknowledgements $\mathrm{We}$ are grateful to all local physicians, the nursing staff and patients who participated in this study. P. Schuetz was supported by a research grant from the Swiss Foundation for Grants in Biology and Medicine (Schweizerische Stiftung für medizinisch-biologische Stipendien, SSMBS, PASMP3-127684/1). N. I. Shapiro is supported in part by National Institutes of Health grants: HL091757 and GM076659 (NIS).

Duality of interest N. I. Shapiro has received research grants from Hutchinson technologies, Eli Lilly and Inverness Medical. All other authors declare that there is no duality of interest associated with this manuscript.

\section{References}

1. Angus DC, Linde-Zwirble WT, Lidicker J, Clermont G, Carcillo J, Pinsky MR (2001) Epidemiology of severe sepsis in the United States: analysis of incidence, outcome, and associated costs of care. Crit Care Med 29:1303-1310 
2. Lee WL, Slutsky AS (2010) Sepsis and endothelial permeability. N Engl J Med 363:689-691

3. Friedman G, Silva E, Vincent JL (1998) Has the mortality of septic shock changed with time. Crit Care Med 26:2078-2086

4. Esper AM, Moss M, Lewis CA, Nisbet R, Mannino DM, Martin GS (2006) The role of infection and comorbidity: factors that influence disparities in sepsis. Crit Care Med 34:2576-2582

5. Benfield T, Jensen JS, Nordestgaard BG (2007) Influence of diabetes and hyperglycaemia on infectious disease hospitalisation and outcome. Diabetologia 50:549-554

6. Stegenga ME, Vincent JL, Vail GM, Xie J, Haney DJ, Williams MD et al (2010) Diabetes does not alter mortality or hemostatic and inflammatory responses in patients with severe sepsis. Crit Care Med 38:539-545

7. Vincent JL, Preiser JC, Sprung CL, Moreno R, Sakr Y (2010) Insulin-treated diabetes is not associated with increased mortality in critically ill patients. Crit Care 14:R12

8. Aird WC (2003) The role of the endothelium in severe sepsis and multiple organ dysfunction syndrome. Blood 101:3765-3777

9. Reinhart K, Bayer O, Brunkhorst F, Meisner M (2002) Markers of endothelial damage in organ dysfunction and sepsis. Crit Care Med 30(Suppl):S302-S312

10. London NR, Zhu W, Bozza FA, Smith MC, Greif DM, Sorensen LK et al (2010) Targeting Robo4-dependent Slit signaling to survive the cytokine storm in sepsis and influenza. Sci Transl Med 2:23ra19

11. Aird WC (2008) Endothelium in health and disease. Pharmacol Rep 60:139-143

12. Shapiro NI, Schuetz P, Kiichiro Y, Sorasaki M, Jones AE, Trzeciak S et al (2010) The association of endothelial cell signaling, severity of illness, and organ dysfunction in sepsis. Crit Care 14:R182

13. Dandona P, Aljada A, Chaudhuri A, Mohanty P (2004) Endothelial dysfunction, inflammation and diabetes. Rev Endocr Metab Disord 5:189-197

14. Aljada A (2003) Endothelium, inflammation, and diabetes. Metab Syndr Relat Disord 1:3-21

15. Gomez JM, Vila R, Catalina P, Soler J, Badimon L, Sahun M (2008) The markers of inflammation and endothelial dysfunction in correlation with glycated haemoglobin are present in type 2 diabetes mellitus patients but not in their relatives. Glycoconj $\mathrm{J}$ 25:573-579

16. Gimbrone MA Jr, Bevilacqua MP, Cybulsky MI (1990) Endothelial-dependent mechanisms of leukocyte adhesion in inflammation and atherosclerosis. Ann NY Acad Sci 598:77-85

17. el-Mesallamy H, Suwailem S, Hamdy N (2007) Evaluation of Creactive protein, endothelin-1, adhesion molecule(s), and lipids as inflammatory markers in type 2 diabetes mellitus patients. Mediat Inflamm 2007:73635

18. Panahloo A, Yudkin JS (1997) Diminished fibrinolysis in diabetes mellitus and its implication for diabetic vascular disease. J Cardiovasc Risk 4:91-99

19. Kruszynska YT, Yu JG, Olefsky JM, Sobel BE (2000) Effects of troglitazone on blood concentrations of plasminogen activator inhibitor 1 in patients with type 2 diabetes and in lean and obese normal subjects. Diabetes 49:633-639

20. Kim NH, Oh JH, Seo JA et al (2005) Vascular endothelial growth factor (VEGF) and soluble VEGF receptor FLT-1 in diabetic nephropathy. Kidney Int 67:167-177

21. Bone R (1992) American College of Chest Physicians/Society of Critical Care Medicine Consensus Conference: definitions for sepsis and organ failure and guidelines for the use of innovative therapies in sepsis. Crit Care Med 20:864-874

22. Shapiro N, Howell MD, Bates DW, Angus DC, Ngo L, Talmor D (2006) The association of sepsis syndrome and organ dysfunction with mortality in emergency department patients with suspected infection. Ann Emerg Med 48:583-590

23. Levy MM, Fink MP, Marshall JC, Abraham E, Angus D, Cook D et al (2003) $2001 \mathrm{SCCM} / \mathrm{ESICM} / \mathrm{ACCP} / \mathrm{ATS} / \mathrm{SIS}$ International Sepsis Definitions Conference. Intensive Care Med 29:530-538

24. Vincent J, Moreno R, Takala J, Willatts S, de Mendonca A, Bruining $\mathrm{H}$ et al (1996) The SOFA (sepsis-related organ failure assessment) score to describe organ dysfunction/failure. Intensive Care Med 22:707-710

25. Knaus WA, Draper EA, Wagner DP, Zimmerman JE (1985) APACHE II: a severity of disease classification system. Crit Care Med 13:818-829

26. Matsumoto K, Sera Y, Miyake S, Ueki Y (2002) Serum levels of adhesion molecules correlate with insulin resistance. Atherosclerosis 161:243-244

27. Meigs JB, Hu FB, Rifai N, Manson JE (2004) Biomarkers of endothelial dysfunction and risk of type 2 diabetes mellitus. JAMA 291:1978-1986

28. Hwang SJ, Ballantyne CM, Sharrett AR, Smith LC, Davis CE, Gotto AM Jr et al (1997) Circulating adhesion molecules VCAM1, ICAM-1, and E-selectin in carotid atherosclerosis and incident coronary heart disease cases: the Atherosclerosis Risk In Communities (ARIC) Study. Circulation 96:4219-4225

29. Blann AD, Belgore FM, McCollum CN, Silverman S, Lip PL, Lip GY (2002) Vascular endothelial growth factor and its receptor, Flt1 , in the plasma of patients with coronary or peripheral atherosclerosis, or type II diabetes. Clin Sci 102:187-194

30. Andreasen AS, Pedersen-Skovsgaard T, Berg RM, Svendsen KD, Feldt-Rasmussen B, Pedersen BK et al (2010) Type 2 diabetes mellitus is associated with impaired cytokine response and adhesion molecule expression in human endotoxemia. Intensive Care Med 36:1548-1555

31. Sarnak MJ, Jaber BL (2000) Mortality caused by sepsis in patients with end-stage renal disease compared with the general population. Kidney Int 58:1758-1764

32. Kornum JB, Thomsen RW, Riis A, Lervang HH, Schonheyder HC, Sorensen HT (2007) Type 2 diabetes and pneumonia outcomes: a population-based cohort study. Diab Care 30:2251-2257

33. Esper AM, Moss M, Martin GS (2009) The effect of diabetes mellitus on organ dysfunction with sepsis: an epidemiological study. Crit Care 13:R18

34. Thomsen RW, Hundborg HH, Lervang HH, Johnsen SP, Schonheyder HC, Sorensen HT (2005) Diabetes mellitus as a risk and prognostic factor for community-acquired bacteremia due to enterobacteria: a 10-year, population-based study among adults. Clin Infect Dis 40:628-631

35. Tsai CL, Lee CC, Ma MH et al (2007) Impact of diabetes on mortality among patients with community-acquired bacteremia. $\mathrm{J}$ Infect 55:27-33

36. Leone M, Boutiere B, Camoin-Jau L et al (2002) Systemic endothelial activation is greater in septic than in traumatichemorrhagic shock but does not correlate with endothelial activation in skin biopsies. Crit Care Med 30:808-814

37. Bierhaus A, Nawroth PP (2009) Multiple levels of regulation determine the role of the receptor for AGE (RAGE) as common soil in inflammation, immune responses and diabetes mellitus and its complications. Diabetologia 52:2251-2263 